\title{
Angelica keiskei (Ashitaba) has potential as an antithrombotic health food
}

\author{
${ }^{1}$ Ohkura, N., ${ }^{2}$ Taniguchi, M., ${ }^{3}$ Oishi, K., ${ }^{4}$ Inoue, K. and ${ }^{5}$ Ohta, M. \\ ${ }^{1}$ Laboratory of Host Defense, Department of Medical and Pharmaceutical Sciences, School of Pharma- \\ Sciences, Teikyo University, Japan \\ ${ }^{2}$ Department of Natural Products Research, Osaka Medical and Pharmaceutical University, Japan \\ ${ }^{3}$ Healthy Food Science Research Group, Cellular and Molecular Biotechnology Research Institute, National \\ Institute of Advanced Industrial Science and Technology (AIST), Japan \\ ${ }^{4}$ Japan Bio Science Laboratory Co. Ltd., Osaka, Japan \\ ${ }^{5}$ Research Institute of Production Development, Kyoto, Japan
}

\section{Article history:}

Received: 1 March 2021

Received in revised form: 11

April 2021

Accepted: 17 June 2021

Available Online: 6 March

2022

\section{Keywords:}

Thrombosis,

Inflammation,

Chalcone,

Platelet,

PAI-1

DOI:

https://doi.org/10.26656/fr.2017.6(2).121

\begin{abstract}
Angelica keiskei (Ashitaba) is a large perennial herb that is native to the Pacific coast of Japan. It has recently become popular as a healthy food in Asian countries because it might have various physiological benefits including antithrombotic properties. Most studies of the bioactive constituents from Ashitaba have focused on the activities of the major chalcones, xanthoangelol and 4-hydroxyderricin. However, other chalcones, flavanones and coumarins have also been isolated from Ashitaba, precisely characterized, and investigated in vivo. Platelets play a key role in haemostasis and wound healing processes. Dysregulated platelet activity is associated with the progression of platelet aggregation and decreased venous blood flow, which results in thrombotic diseases. A minor chalcone, xanthoangelol $\mathrm{E}$, inhibits $\mathrm{TXB}_{2}$ synthesis in rabbit platelets, which seems to be the source of the belief that Ashitaba has antithrombotic properties. However, recent data showed that xanthoangelol and 4-hydroxyderricin inhibited the aggregation of rabbit platelets. Platelet aggregation stimulated by collagen was also inhibited in whole blood incubated with Xanthoangelol or 4-hydroxyderricin. Plasminogen activator inhibitor-1 is the primary physiological inhibitor of tissue type plasminogen activator, a key protease of the fibrinolytic system. An increase in plasma of this inhibitor is associated with thrombotic conditions. Ashitaba yellow exudate inhibited the elevation of plasma plasminogen activator inhibitor-1 in mice induced by obesity or chronic low-grade inflammation. These studies showed the yellow exudate from stem cuttings and chalcones isolated from Ashitaba roots and leaves might have antithrombotic activity. This article reviews the possible antithrombotic properties of Ashitaba.
\end{abstract}

\section{Introduction}

Metabolic syndromes such as diabetes, hypertension, obesity, and dyslipidaemia are associated with an increased risk of thrombotic diseases caused by the formation of blood clots inside blood vessels that obstruct blood flow through the circulatory system. Myocardial and cerebral infarctions and venous thromboembolism are classified as thrombotic diseases, the prevalence of which is increasing along with that of metabolic syndrome (Grundy et al., 2005; Goldhaber, 2010; Previtali et al., 2011). Elevated blood sugars and lipids decrease blood fluidity, which in turn lowers the threshold of platelet activation, increases amounts and activities of blood coagulation factors, and suppresses the blood fibrinolytic system in metabolic syndrome; all of these changes are associated with thrombotic diseases (Russo, 2012).

The risk of thrombotic diseases due to lifestyle habits can be reduced not only by pharmacotherapeutics but also by exercise and consuming dietary supplements that might help blood and blood vessels regain healthy status. Although many health foods are considered effective against thrombotic diseases, the science behind the ingredients that might prevent blood clots should be investigated in more detail. We have recently focused on health foods that might prevent thrombosis, and suggest 
that Ashitaba is an antithrombotic health food. Here, we introduce the possibility that ingesting Ashitaba or its constituents prevents thrombotic diseases.

\section{Ashitaba and its unique components, chalcones}

Ashitaba (Angelica keiskei Koidzumi) is a large perennial herb of the Apiaceae genus Angelica (Figure 1). Ashitaba is native to the Pacific coast of Japan (Izu Islands and the Izu, Bōso, and Miura peninsulas), and it is now cultivated in Southeast Asia and the Korean peninsula. Ashitaba is the common Japanese name for the Angelica keiskei cultivar Koidzumi, and the English translation of it is "tomorrow's leaf". This description reflects the vitality of Ashitaba, which can regenerate within one day of cutting (Baba, 2013). Ashitaba has been consumed as a vegetable and used as folk medicine since ancient times. The leaves, stems, and roots of Ashitaba are rich in nutrients such as vitamin A, vitamin $\mathrm{K}$, and dietary fibre, as well as various unique polyphenols such as chalcones and coumarin. Powdered Ashitaba leaves, roots, and yellow exudates have become popular as societies have become increasingly healthconscious. Various compounds in Ashitaba such as chalcones, flavanones and coumarin have been isolated and their structures and bioactivity have been investigated (Caesar et al., 2016; Kil et al., 2017). The structures of 10 chalcones isolated from Ashitaba have been determined (Hata and Kozawa, 1961; Kozawa et al., 1977; Kozawa et al., 1978; Baba, Nakata, Taniguchi et al., 1990, Baba, Kito, Yoneda et al., 1990; Nakata et al., 1999; Figure 2). The chalcones, xanthoangelol (XA) and 4-hydroxydelicin (4-HD) account for $>90 \%$ of all chalcones identified in Ashitaba. The remaining chalcones comprise trace amounts of xanthoangelols B, C, D, E, F, G, and H, and isobavachalcones. Chalcones are found in leaves, stems, roots and yellow exudates from cut stems. Ashitaba chalcone has a long history of human use as a dietary supplement with claims of multiple health benefits. Previously published animal studies were without toxic effects (Maronpot, 2015).

Studies of the physiological activities of Ashitaba were initiated based on the folklore of the Izu Islands (Baba, 2013). The initial discovery was that Ashitaba chalcones might inhibit gastric acid secretion, and have antitumor and antibacterial activities (Murakami et al., 1990; Inamori et al., 1991; Okuyama et al., 1991). Thereafter, various physiological activities of the major chalcones, XA and 4-HD were determined (Murakami et al., 1990; Inamori et al., 1991; Kimura et al., 2003; Kimura et al., 2004; Ogawa et al., 2005; Ogawa et al., 2007; Shin et al., 2011). After finding that Ashitaba can suppress high blood glucose and exert anti-obesity effects in mice (Enoki et al., 2007), it attracted attention as a health food that might improve lifestyle diseases such as obesity and diabetes, and animal and clinical studies were conducted (Zhang et al., 2015; Ohnishi et al., 2017, Kalman et al., 2018; Ohta et al., 2019; Oh et al., 2019; Zhang et al., 2019). Accumulating evidence supports the notion that Ashitaba could be a useful health food or dietary supplement.

A

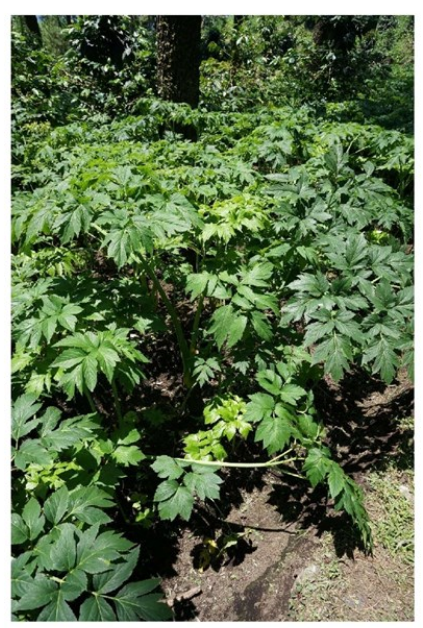

B

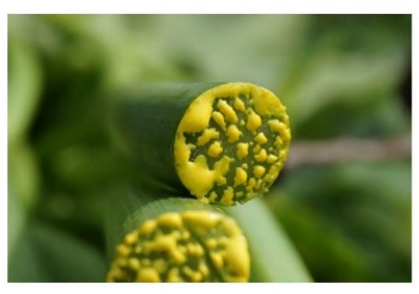

Figure 1. A: Angelica keiskei Koidzumi (Ashitaba), B: Yellow exudate from stem cuttings

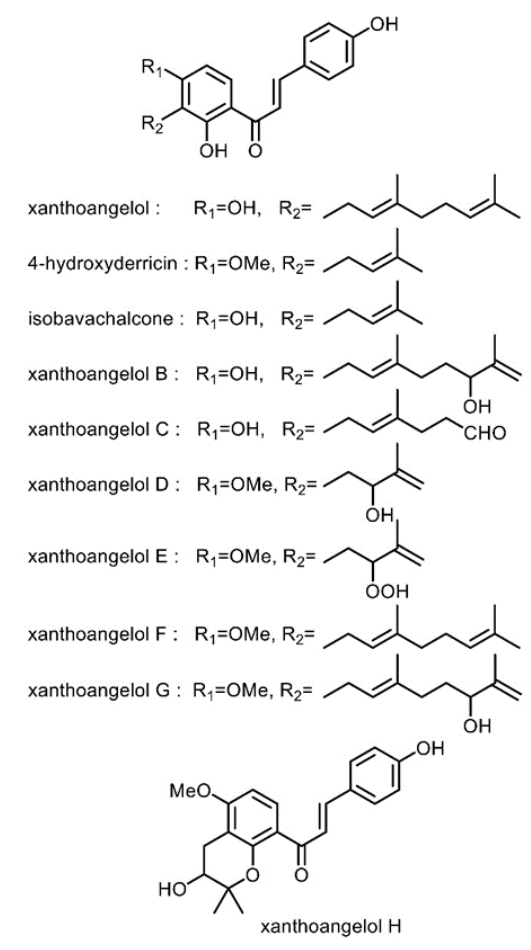

Figure 2. Ashitaba chalcones 
3. How the belief that Ashitaba has antithrombotic properties developed

The haemostatic system comprises platelets, coagulation factors and the fibrinolytic system. Antithrombotic activity generally refers to antiplatelet action and anticoagulant action induced by plasma coagulation factors. In general, antithrombotic agents comprise anticoagulants that inhibit the coagulation cascade of blood coagulation factors that interfere with further clot expansion, and antiplatelet substances that decrease platelet aggregation and inhibit platelet thrombus formation. Therefore, to determine whether candidate substances exert anti-thrombotic effects, their actions on the blood coagulation system and platelets, and the fibrinolytic system should be investigated not only in vitro but also in vivo. The first study of possible antithrombotic effects showed that isolated xanthoangelol $\mathrm{E}$, trace amounts of which are found in Ashitaba, inhibits thromboxane $\mathrm{B}_{2}\left(\mathrm{TXB}_{2}\right)$ synthesis in vitro (Fujita et al., 1992). However, the authors did not describe whether xanthoangelol $\mathrm{E}$ inhibits platelet aggregation. Fujita et al. (1992) found that xanthoangelol $\mathrm{E}$ inhibited the production of $\mathrm{TXB}_{2}$ and 12-hydroxy5,8,10-heptadecatrienoic acid from exogenous arachidonic acid in rabbit platelets. However, they did not prove that xanthoangelol $\mathrm{E}$ inhibits platelet aggregation. Hence, whether or not xanthoangelol $\mathrm{E}$ inhibits platelet aggregation and exerts antithrombotic activity in vivo remains unclear. Regardless, this finding seems to be the source of the popular belief that Ashitaba has antithrombotic activity (Ohkura et al., 2018).

\section{Effects of Ashitaba chalcones on platelets}

Platelets play a key role in haemostasis and wound healing processes to maintain the integrity of the circulatory system. Dysregulated platelet activity is associated with the progression of platelet aggregation and decreased venous blood flow, which results in thrombotic diseases (George, 2000). Son and coworkers. assessed the effects of XA and 4-HD on the aggregation of rabbit washed platelets. They found that $\mathrm{XA}$ and 4-HD, the main components of Ashitaba chalcones, inhibited platelet aggregation induced by stimulation with collagen, platelet-activating factor (PAF) and phorbol 12-myristate 13-acetate, but not by thrombin (Son et al., 2014). This indicated that XA and 4 -HD are more likely to inhibit the phosphoinositide phospholipase C gamma (PLC $\gamma$ )-, rather than PLC $\beta$ related activation pathway in platelets (Son et al., 2014).

Although Son and co-workers investigated the effects of chalcones on isolated platelets, we assessed these effects on whole blood platelet aggregation. Our findings also showed that Ashitaba chalcones inhibit platelet aggregation (Ohkura et al., 2016). Platelet aggregation stimulated by collagen was inhibited in bovine whole blood incubated with XA or 4-HD. Tail bleeding in mice is primarily due to platelet plug formation as a result of platelet aggregation, and platelet function in vitro is generally measured as tail bleeding time (Day et al., 2004). The haemostatic function of platelets in vivo can be determined by measuring the amount of time required to stop bleeding from a small wound in mouse tails (tail bleeding assay; Beviglia et al., 1993). Therefore, we investigated whether Ashitaba yellow exudate could suppress mouse tail bleeding to determine platelet function in vivo (Ohkura et al., 2016). Mice were orally administered with Ashitaba yellow exudate once daily for 7 days, then lipopolysaccharide (LPS) was intraperitoneally administered to induce a thrombotic tendency. The tail tip was cut with a sharp knife under complete anaesthesia, and we measured the amount of time required to stop bleeding. We found that bleeding stopped sooner in LPS-stimulated than in control mice. This is because the LPS induced platelet aggregation, which shortened the amount of time needed to achieve haemostasis (MacIntyre et al., 1977). In contrast, the elapsed time to haemostasis recovered to the normal level in mice given oral Ashitaba yellow exudate (Ohkura et al., 2016). This indicated that the oral Ashitaba yellow exudate inhibits platelet activation in vivo. As described above, Ashitaba yellow exudate contains not only XA and 4-HD but also $>10$ other chalcones with slightly different structures from those of $\mathrm{XA}$ and 4-HD, and many other compounds. Therefore, we investigated the effects of various isolated Ashitaba chalcones on mouse tail bleeding (Ohkura et al., 2016). We injected XA, 4-HD, XB, XD, XE and XF into the mouse peritoneal cavity and found that only XA significantly recovered tail bleeding. Therefore, structural differences in the side chains of chalcones are important for their bioactivities. The hydrocarbon side chain of Ashitaba chalcones plays an important role and small modifications to this chain, or the addition of a small functional group to the A ring influences the antiplatelet activities of these chalcones. Only XA was effective in terms of tail bleeding time and platelet aggregation in mice. In contrast, both XA and 4-HD inhibited bovine and human platelet aggregation. The removal of prenylation in 4-HD decreases hydrophobicity and thus might decrease mouse platelet translocation or interactions. Regardless, considering that $>90 \%$ of the chalcones in Ashitaba yellow exudate are $\mathrm{XA}$ and 4-HD, the inhibition of platelet function seems to be the main effect of these two Ashitaba chalcones. 
5. Effects of Ashitaba chalcones on coagulation factors in plasma

Ashitaba chalcones were examined for their effect on the coagulation pathway by measuring prothrombin time (PT) and activated partial thromboplastin time (APTT) that reflect the amount of time required for plasma to coagulate after adding a coagulation initiator (Ohkura et al., 2011). Prothrombin time and APTT indicate the integrity of coagulation factors in the common system, and in the extrinsic and intrinsic systems, respectively. These tests are clinically applied to screen coagulation factor deficiencies. These tests are defined as the time (seconds) needed to clot plasma upon addition of coagulation triggers, such as tissue factor in complex with phospholipids and calcium chloride (in the PT), or negatively charged phospholipids-activators and calcium chloride (in the APTT). They are considered global coagulation tests sensitive to the amounts of coagulation factors. Prothrombin time and APTT are both sensitive to factor $\mathrm{X}, \mathrm{V}, \mathrm{II}$ and fibrinogen, whereas the PT is only sensitive to factor VII amounts and the APTT is sensitive to factor XII, XI, IX and VIII amounts. Furthermore, the PT and APTT are variably sensitive to the presence of antithrombotic agents directed against specific coagulation factors. These assays are also used to explore novel anticoagulant agents (Chee, 2014).

Adding coagulation inhibitors to plasma prolongs PT and/or APTT by inhibiting coagulation factors involved in the extrinsic and intrinsic coagulation pathways, respectively. Adding those that prolong both PT and APTT inhibit coagulation factors involved in a common pathway. We added XA or 4-HD to human plasma followed by each coagulation initiator, and compared PT or APTT with those of control samples without XA and 4-HD. Coagulation times did not differ between the samples and controls (Ohkura et al., 2011), indicating that neither XA nor 4-HD inhibited the plasma coagulation system according to these assays.

\section{Effects of Ashitaba chalcones on fibrinolysis}

Fibrinolysis is a highly regulated enzymatic process that prevents unnecessary accumulation of intravascular fibrin and enables thrombus removal. The fibrinolytic system removes fibrin from the vascular system, thus preventing haemostatic clot enlargement and vessel occlusion (Chapin et al., 2015). Like the coagulation cascade, fibrinolysis is tightly controlled by a series of proteases, protease inhibitors, and receptors. Plasmin is the key fibrinolytic protease, and it is activated from plasminogen by either tissue plasminogen activator (tPA) or urokinase ( $\mathrm{UPA})$ which are primary serine proteases (Chapin et al., 2015). Fibrinolysis is regulated by these two PA and its crucial inhibitor, plasminogen activator inhibitor-1 (PAI-1).
Liver, adipose tissues, and muscle, bone, and hematopoietic cells express PAI-1 that inhibits thrombolysis in blood fibrinolytic reactions (Van Den Craen et al., 2012). Thus, high plasma PAI-1 levels are causally related to attenuated fibrinolysis and increased risk for thrombosis. Since plasma PAI-1 levels are highly elevated under various pathological conditions including infection and inflammation, their fibrinolytic potential in plasma and on vascular endothelial cells is readily suppressed and persistent blood clots lead to thrombosis (Van Den Craen et al., 2012). Other than this, plasma PAI-1 is elevated in patients with metabolic syndrome including obesity and diabetes. Thus, PAI-1 might be associated with a thrombotic tendency in this syndrome (Alessi et al., 2011). Since adipose tissues produce large amounts of PAI-1, the elevated plasma PAI-1 in obesity is thought to originate from these tissues. Adipocytes synthesize PAI-1, and plasma PAI-1 levels are increased in obesity and reduced by weight loss (Cesari et al., 2010). That is, a link between PAI-1 and metabolic syndrome has been established and elevated plasma PAI1 levels are now considered a true component of the thrombotic tendency in this syndrome (Alessi et al., 2011).

We recently analysed the effects of Ashitaba yellow exudate on enhanced PAI-1 levels in obese diabetic mice (Ohta et al., 2019). Intake of Ashitaba yellow exudate significantly decreased food efficiency and plasma PAI-1 in obese diabetic mice but did not affect lean control mice. Ashitaba yellow exudate also decreased some parameters in plasma, such as glucose, insulin, tumour necrosis factor-alpha (TNF- $\alpha$ ) and gains in body, subcutaneous, and mesenteric fat weight in TSOD mice but had little effect on these parameters in TSNO mice. These findings suggested that Ashitaba yellow exudate decreased plasma PAI-1 levels by suppressing both the adipose tissue retention of PAI-1 protein and liver PAI-1 production in obese diabetic mice (Ohta et al., 2019). Thus, supplementing diets with Ashitaba might help to prevent or alleviate the risk of thrombotic diseases, and suppress dysfunctional metabolic states in obese individuals.

Chronic low-grade inflammation has been linked to the progression of obesity and related diseases (Kimura et al., 2003; Kimura et al., 2004). Therefore, controlling PAI-1 elevation associated with chronic low-grade inflammation is thought to lead to the prevention of thrombosis caused by not only inflammation but also lifestyle-related diseases. We used low-grade inflammation to induce a thrombotic tendency in a mouse model induced by extremely low levels of lipopolysaccharide (LPS) and found that oral Ashitaba yellow exudate inhibited plasma PAI-1 elevation 
(Ohkura et al., 2011). Ashitaba also suppressed PAI-1 production in the liver, heart, and adipose tissues in this model (Ohkura et al., 2011) and in cultured endothelial cells. Human umbilical endothelial cells (HUVEC) stimulated with inflammatory cytokines such as TNF- $\alpha$ increase the amount of PAI-1 released into the medium. We cultured HUVEC with various Ashitaba chalcones then stimulated the cells with TNF- $\alpha$. We found that XA, $\mathrm{XB}$ and $\mathrm{XD}$ inhibited PAI-1 release into the medium induced by TNF- $\alpha$ (Ohkura et al., 2011).

However, as described above, $\geq 90 \%$ of chalcones in Ashitaba comprise XA and 4-HD. We later discovered that 4-HD does not inhibit, whereas XA suppresses PAI1 production by the HUVEC cell line, EA.hy926, (Ohkura et al., 2015). Considering these findings, the inhibitory action of PAI-1 production by oral Ashitaba yellow exudate is thought to be due to XA action. As described above, Ashitaba yellow exudate suppressed PAI-1 production in the heart, liver, and adipose tissues, and Nakamura et al. (2012) showed that XA and 4-HD are rapidly absorbed and distributed to various tissues. Thus, Ashitaba chalcones inhibit PAI-1 production in adipocytes and hepatocytes.

\section{Conclusion}

Dysregulated platelet activity is associated with the progression of platelet aggregation which results in thrombotic diseases. Two major Ashitaba chalcone, xanthoangelol and 4-hydroxyderricin, inhibited the platelet aggregation induced by various stimulants. Xanthoangelol was effective in terms of tail bleeding time that reflect platelet activity in vitro in mice and platelet aggregation in mice whole blood. On the other hand, Ashitaba yellow exudate inhibited the elevation of PAI-1, the primary physiological inhibitor of tissue type plasminogen activator, in mice induced by obesity or chronic low-grade inflammation. Whether intake of Ashitaba or Ashitaba chalcones confers health benefits upon humans remains to be elucidated. However, current findings indicate that dietary Ashitaba might help to prevent thrombotic diseases and maintain good health.

\section{Conflict of interest}

The authors have no conflicts of interest to declare. The authors alone are responsible for the content and writing of the article.

\section{References}

Alessi, M.C., Nicaud, V., Scroyen, I., Lange, C., Saut, N., Fumeron, F., Marre, M., Lantieri, O., FontaineBisson, B., Juhan-Vague, I., Balkau, B., Tregouet, D.A., Morange, P.E. and DESIR Study Group
(2011). Association of vitronectin and plasminogen activator inhibitor-1 levels with the risk of metabolic syndrome and type 2 diabetes mellitus. Results from the D.E.S.I.R. prospective cohort. Thrombosis Haemostasis, 106(3), 416-422. https:// doi.org/10.1160/TH11-03-0179

Baba, K. (2013). Studies on the Chemical Components and Biological Activities of Angelica keiskei Koidzumi. Bulletin of Osaka University of Pharmaceutical Sciences, 7, 55-87.

Baba, K., Nakata, K., Taniguchi, M., Kido, T. and Kozawa, M. (1990). Chalcones from Angelica keiskei. Phytochemistry, 29(12), 3907-3910. https:// doi.org/10.1016/0031-9422(90)85357-L

Baba, K., Kito, T., Yoneda, Y., Taniguchi, M. and Kozawa, M. (1990). Chemical Components of Angelica keiskei KOIDZUMI (V): Components of the Fruits, and Comparison of Coumarins and Chalcones in the Fruits, Roots and the Leaves. The Japanese Journal of Pharmacognosy, 44(3), 235239.

Beviglia, L., Poggi, A., Rossi, C., McLane, M.A., Calabrese, R., Scanziani, E., Cook, J.J. and Niewiarowski, S. (1993). Mouse antithrombotic assay. Inhibition of platelet thromboembolism by disintegrins. Thrombosis Research, 71(4), 301-315. https://doi.org/10.1016/0049-3848(93)90199-X

Caesar, L.K. and Cech, N.B. (2016), A Review of the Medicinal Uses and Pharmacology of Ashitaba. Planta Medica, 82(14), 1236-1245. https:// doi.org/10.1055/s-0042-110496

Cesari, M., Pahor, M. and Incalzi, R.A. (2010), Plasminogen Activator Inhibitor-1 (PAI-1): A Key Factor Linking Fibrinolysis and Age-Related Subclinical and Clinical Conditions. Cardiovascular Therapeutics, 28(5), e72-91. https://doi.org/10.1111/ j.1755-5922.2010.00171.x

Chapin, J.C. and Hajjar, K.A. (2015). Fibrinolysis and the control of blood coagulation. Blood Review, 29 (1), 17-24. https://doi.org/10.1016/j.blre.2014.09.003

Chee, Y.L. (2014). Coagulation. The Journal of the Royal College of Physicians of Edinburgh, 44(1), 4245. https://doi.org/10.4997/JRCPE.2014.110

Day, S.M., Reeve, J.L., Myers, D.D. and Fay, W.P. (2004). Murine thrombosis models. Thrombosis Haemostasis, 92(3), 486-494. https:// doi.org/10.1055/s-0037-1613739

Enoki, T., Ohnogi, H., Nagamine, K., Kudo, Y., Sugiyama. K., Tanabe, M., Kobayashi, E., Sagawa, H. and Kato, I. (2007). Antidiabetic activities of chalcones isolated from a Japanese Herb, Angelica keiskei. Journal of Agricultural and Food Chemistry, 
55(15), 6013-6017. https://doi.org/10.1021/ jf070720q

Fujita, T., Sakuma, S., Sumiya, T., Nishida, H., Fujimoto, Y., Baba, K. and Kozawa, M. (1992). The effects of xanthoangelol $\mathrm{E}$ on arachidonic acid metabolism in the gastric antral mucosa and platelet of the rabbit. Research Communications in Chemical Pathology and Pharmacology, 77(2), 227-240.

George, J.N. (2000). Platelets. Lancet (London, England), 355(9214), 1531-1539. https:// doi.org/10.1016/S0140-6736(00)02175-9

Goldhaber, S.Z. (2010). Risk factors for venous thromboembolism. Journal of the American College of Cardiology, 56(1), 1-7. https://doi.org/10.1016/ j.jacc.2010.01.057

Grundy, S.M., Cleeman, J.I., Daniels, S.R., Donato, K.A., Eckel, R.H., Franklin, B.A., Gordon, D.J., Krauss, R.M., Savage, P.J., Smith, S.C.Jr, Spertus, J.A. and Costa, F. (2005). Diagnosis and management of the metabolic syndrome: an American Heart Association/National Heart, Lung, and Blood Institute Scientific Statement. Circulation, 112(17), 2735-2752. https://doi.org/10.1161/ CIRCULATIONAHA.105.169404

Inamori, Y., Baba, K., Tsujibo, H., Taniguchi, M., Nakata, K. and Kozawa, M. (1991). Antibacterial activity of two chalcones, xanthoangelol and 4hydroxyderricin, isolated from the root of Angelica keiskei KOIDZUMI. Chemical and Pharmaceutical Bulletin, 39(6), 1604-1605. https://doi.org/10.1248/ cpb.39.1604

Kil, Y.S., Pham, S.T., Seo, E.K. and Jafari, M. (2017). Angelica keiskei, an emerging medicinal herb with various bioactive constituents and biological activities. Archives of Pharmacal Research, 40(6), 655-675. https://doi.org/10.1007/s12272-017-0892-3

Kimura, Y. and Baba, K. (2003) Antitumor and antimetastatic activities of Angelica keiskei roots, part 1: Isolation of an active substance, xanthoangelol. International Journal of Cancer, 106 (3), 429-437. https://doi.org/10.1002/ijc.11256

Kimura, Y, Taniguchi, M. and Baba, K (2004). Antitumor and antimetastatic activities of 4hydroxyderricin isolated from Angelica keiskei roots. Planta Medica, 70(3), 211-219. https:// doi.org/10.1002/ijc.11256

Kozawa, M., Morita, N., Baba, K. and Hata, K. (1977). The structure of xanthoangelol, a new chalcone from the roots of Angelica keiskei KOIDZUMI (Umbelliferae). Chemical and Pharmaceutical Bulletin, 25(3), 515-516. https://doi.org/10.1248/ cpb. 25.515
Kozawa, M., Morita, N., Baba, K., Hata, K. (1978) Chemical Components of the Roots of Angelica keiskei KOIDZUMI. II. The Structure of the Chalcone Derivatives. Yakugaku Zasshi, 98(2), 210214. https://doi.org/10.1248/yakushi1947.98.2_210

Hata, K. and Kozawa, M. (1961). Pharmacognosy study of Umbelliferae plants $18^{\text {th }}$ report. Yakugaku Zasshi, 81, 1647-1649. https://doi.org/10.1248/ yakushi1947.81.11_1647 [In Japanese].

Kalman., D.S., Hewlings, S. and Hackel, V. (2018) A study to evaluate chalcurb ${ }^{\circledR}$ a standardized powder derived from the sap of the Angelica keiskei (Ashitaba) on markers of health in adults with metabolic syndrome. Advances in Obesity Weight Management and Control, 8(4), 203-208. https:// doi.org/10.15406/aowmc.2018.08.00244

MacIntyre, D.E., Allen., A.P., Thorne, K.J., Glauert, A.M. and Gordon, J.L. (1977) Endotoxin-induced platelet aggregation and secretion. I. Morphological changes and pharmacological effects. Journal of Cell Science, 28(1), 211-223. https://doi.org/10.1242/ jcs.28.1.211

Maronpot, R.R. (2015) Toxicological assessment of Ashitaba Chalcone. Food and Chemical Toxicology, 77, 111-119. https://doi.org/10.1016/ j.fct.2014.12.021

Murakami, S., Kijima, H., Isobe, Y., Muramatsu, M., Aihara, H., Otomo, S., Baba, K. and Kozawa, M. (1990). Inhibition of gastric $\mathrm{H}+, \mathrm{K}(+)$-ATPase by chalcone derivatives, xanthoangelol and 4hydroxyderricin, from Angelica keiskei Koidzumi. The Journal of Pharmacy and Pharmacology, 42 (10), 723-726. https://doi.org/10.1111/j.20427158.1990.tb06568.x

Nakata, K., Taniguchi, M. and Baba, K. (1999). Three chalcones from Angelica keiskei. Natural Medicines, 53(6), 329-332.

Nakamura, T., Tokushima, T., Kawabata, K., Yamamoto, N., Miyamoto, M. and Ashida, H. (2012). Absorption and metabolism of 4hydroxyderricin and xanthoangelol after oral administration of Angelica keiskei (Ashitaba) extract in mice. Archives of Biochemistry and Biophysics, 521(1-2), 71-76. https://doi.org/10.1016/ j.abb.2012.03.013

Ogawa, H., Okada, Y., Kamisako, T. and Baba, K. (2007) Beneficial effect of xanthoangelol, a chalcone compound from Angelica keiskei, on lipid metabolism in stroke-prone spontaneously hypertensive rats. Clinical and Experimental Pharmacology and Physiology, 34(3), 238-243. https://doi.org/10.1111/j.1440-1681.2007.04578.x 
Ogawa, H., Ohno, M. and Baba, K. (2005) Hypotensive and lipid regulatory actions of 4-hydroxyderricin, a chalcone from Angelica keiskei, in stroke-prone spontaneously hypertensive rats. Clinical and Experimental Pharmacology and Physiology, 32(12), 19-23. https://doi.org/10.1111/j.14401681.2005.04147.x

Oh, H.A., Lee, H., Park, S.Y., Lim, Y., Kwon, O., Kim, J.Y., Kim, D. and Jung, B.H. (2019). Analysis of plasma metabolic profiling and evaluation of the effect of the intake of Angelica keiskei using metabolomics and lipidomics. Journal of Ethnopharmacology, 243, 112058. https:// doi.org/10.1016/j.jep.2019.112058

Ohkura, N., Atsumi, G., Ohnishi, K., Baba, K. and Taniguchi, M. (2018) Possible antithrombotic effects of Angelica keiskei (Ashitaba). Pharmazie, 73(6), 315-317. https://doi.org/10.1691/ph.2018.8370.

Ohkura, N., Ohnishi, K., Taniguchi, M., Nakayama, A., Usuba, Y., Fujita, M., Fujii, A., Ishibashi, K., Baba, $\mathrm{K}$ and Atsumi, G. (2016). Anti-platelet effects of chalcones from Angelica keiskei Koidzumi (Ashitaba) in vivo. Die Pharmazie, 71(11), 651-654. https://doi.org/10.5455/jice.20150910123122

Ohkura, N., Oiwa, H., Ohnishi, K., Taniguchi, M., Baba, K. and Atsumi, G. (2015) Inhibition of plasminogen activator inhibitor-1 release from human endothelial cells by Angelica keiskei Koidzumi (Ashitaba) chalcones is structure-dependent. Journal of Intercultural Ethnopharmacology, 4(4), 355-357. https://doi.org/10.1002/biof.187

Ohkura, N., Nakakuki, Y., Taniguchi, M., Kanai, S., Nakayama, A., Ohnishi, K., Sakata, T., Nohira, T., Matsuda, J., Baba, K. and Atsumi, G. (2011). Xanthoangelols isolated from Angelica keiskei inhibit inflammatory-induced plasminogen activator inhibitor 1 (PAI-1) production. BioFactors (Oxford, England), 37(6), 455-461. https://doi.org/10.1002/ biof. 187

Ohnishi, K. and Hackel, V. (2017). A two part randomized, placebo controlled double blind pilot study to determine the effectt of ashitaba (Angelica keiskei) chalcone powder (chalCurbR) on body weight and visceral fat in slightly obese adults. Advances in Obesity, Weight Management and Control, 7(2), 242-246. https://doi.org/10.15406/ aowmc.2017.07.00189

Ohta, M., Fujinami, A., Oishi, K., Kobayashi, N., Ohnishi, K. and Ohkura, N. (2019) Ashitaba (Angelica Keiskei) Exudate Prevents Increases in Plasminogen Activator Inhibitor-1 Induced by Obesity in Tsumura Suzuki Obese Diabetic Mice. Journal of Dietary Supplements, 16(3), 331-344. https://doi.org/10.1080/19390211.2018.1458366

Okuyama, T., Takata, M., Takayasu, J., Hasegawa, T., Tokuda, H., Nishino, A., Nishino, H. and Iwashima, A. (1991). Anti-tumor-promotion by principles obtained from Angelica keiskei. Planta Medica, 57 (3), 242-246. https://doi.org/10.1055/s-2006-960082

Previtali, E., Bucciarelli, P., Passamonti, S.M. and Martinelli, I. (2011). Risk factors for venous and arterial thrombosis. Blood Transfusion, 9(2), 120138.

Russo, I. (2012). The prothrombotic tendency in metabolic syndrome: focus on the potential mechanisms involved in impaired haemostasis and fibrinolytic balance. Scientifica, 2012, 525374. https://doi.org/10.6064/2012/525374

Son, D.J., Park, Y.O., Yu, C., Lee, S.E. and Park, Y.H. (2014). Bioassay-guided isolation and identification of anti-platelet-active compounds from the root of Ashitaba (Angelica keiskei Koidz.). Natural Product Research, 28(24), 2312-2316. https:// doi.org/10.1080/14786419.2014.931389

Shin, J.E., Choi, E.J., Jin, Q., Jin, H.G. and Woo, E.R. (2011). Chalcones isolated from Angelica keiskei and their inhibition of IL- 6 production in TNF- $\alpha$ stimulated MG-63 cell. Archives of Pharmacal Research, 34(3), 437-442. https://doi.org/10.1007/ s12272-011-0311-0

Van De Craen, B., Declerck, P.J. and Gils, A. (2012). The biochemistry, physiology and pathological roles of PAI-1 and the requirements for PAI-1 inhibition in vivo. Thrombosis Research, 130(4), 576-585. https://doi.org/10.1016/j.thromres.2012.06.023

Zhang, C., Wu, W., Li, X., Xin, X. and Liu, D. (2019). Daily Supplementation with Fresh Angelica keiskei Juice Alleviates High-Fat Diet-Induced Obesity in Mice by Modulating Gut Microbiota Composition. Molecular Nutrition and Food Research, 63(14), e1900248. https://doi.org/10.1002/mnfr.201900248

Zhang, T., Yamashita, Y., Yasuda, M., Yamamoto, N. and Ashida, H. (2015). Ashitaba (Angelica keiskei) extract prevents adiposity in high-fat diet-fed C57BL/6 mice. Food and Function, 6(1), 135-145. https://doi.org/10.1039/C4FO00525B 